\title{
Application of model reduction technique and structural subsection technique on optimal sensor placement of truss structures
}

\author{
Lingling Lư ${ }^{1 \mathrm{a}}$, Xi Wang ${ }^{2 b}$, Lijuan Liao ${ }^{1}$, Yanpeng Wei ${ }^{1}$, \\ Chenguang Huang ${ }^{* 1}$ and Yanchi Liu ${ }^{1 e}$ \\ ${ }^{1}$ Key Laboratory for Mechanics in Fluid Solid Coupling Systems, Institute of Mechanics, \\ Chinese Academy of Sciences, Beijing 100190, China \\ ${ }^{2}$ School of mechanical, Electronic and Control Engineering, Beijing Jiaotong University, Beijing 100044, China
}

(Received April 15, 2014, Revised September 30, 2014, Accepted November 18, 2014)

\begin{abstract}
An optimal sensor placement (OSP) method based on structural subsection technique (SST) and model reduction technique was proposed for modal identification of truss structures, which was conducted using genetic algorithm (GA). The constraints of GA variables were determined by SST in advance. Subsequently, according to model reduction technique, the optimal group of master degrees of freedom and the optimal objective function value were obtained using GA in a case of the given number of sensors. Correspondingly, the optimal number of sensors was determined according to optimal objective function values in cases of the different number of sensors. The proposed method was applied on a scaled jacket offshore platform to get its optimal number of sensors and the corresponding optimal sensor layout. Then modal kinetic energy and modal assurance criterion were adopted to evaluate vibration energy and mode independence property. The experiment was also conducted to verify the effectiveness of the selected optimal sensor layout. The results showed that experimental modes agreed reasonably well with numerical results. Moreover the influence of the proposed method using different optimal algorithms and model reduction technique on optimal results was also compared. The results showed that the influence was very little.
\end{abstract}

Keywords: structural health monitoring; optimal sensor placement; model reduction technique; genetic algorithm

\section{Introduction}

The problem of parameter identification of structural models using measured dynamic data has received much attention over the years because of its importance in structural model updating, structural health monitoring (SHM) and structural control. The quality of information that can be extracted from the data for estimating the model parameters depends on the number and locations

*Corresponding author, Professor, E-mail: huangcg@imech.ac.cn

${ }^{a}$ Ph. D., Student, Email: lulingling@imech.ac.cn

${ }^{\mathrm{b}} \mathrm{Ph}$. D., E-mail: wangxi@bjtu.edu.cn 
of sensors in the structure (Papadimitriou 2004). However, plenty of sensors would result in high cost of data acquisition systems (sensors and supporting instruments) and workload. Meanwhile, accessibility limitations constrain wide distribution of sensors in many cases. On the other hand, structural responses measured at specified sensor positions determine the accuracy of modal parameter identification and are crucial in the consequent model updating and damage quantification. Therefore, how to optimally determine the number of sensors and deploy them so that the response data acquired from those locations will result in the best identification of structural characteristics are challenging tasks (Yi et al. 2011).

Normally, for structures with simple geometry, optimal sensor placement (OSP) was conducted according to experiences of civil engineers. However, for a large scale complicated structure, reliable and efficient methods are needed. Numerous approaches have been widely reported over the years for structural modal identification. Salama et al. (1987) improved the traditional method using engineers' experiences and proposed modal kinetic energy (MKE) to select locations with high MKE. Based on MKE, average MKE (AMKE) and weighted AMKE (WAMKE) were presented (Larson et al. 1994, Chung and Moore 1993). Similar to MKE, the driving point residue (DPR), average DPR (ADPR) and weighted ADPR were also developed (Chung and Moore 1993). Meanwhile, some OSP criterions considering independence of target mode shapes were presented. The effectiveness independence (EFI) method developed by Kammer (1991) was commonly cited. The goal of the method is to select a sensor configuration which can maximize the trace and determinant and minimize the condition number of the Fisher information matrix corresponding to the target modal partitions. EFI-DPR (Imamovic 1998) is a compromise between the EFI and energy approach. Stephan (2012) added a second optimization criterion to EFI and determined an optimal set of sensors by maximizing the norm of a Fisher information matrix and avoiding the redundancy of information between the selected degrees of freedom. Hanis and Hromcik (2012) improved EFI method by modifying criterion to meet two requirements: maximize useful information and minimize spillover of unwanted higher modes simultaneously. The information entropy norm was also used, which was to minimize the uncertainty in the model parameter estimates (Papadimitriou et al. 2000). Subsequently, many improvements were proposed (Papadimitriou 2004, Papadimitriou 2005, Papadimitriou and Lombaert 2012). Ye and Ni (2012) presented an OSP method based on information entropy for the purposed of damage detection. The results show that the optimal sensor layout displays a good performance on damage identification. To guarantee the measured modal vectors are orthogonal, modal assurance criterion (MAC) (Carne and Dohmann 1995) was also used which is an ideal scalar constant relating the causal relationship between two modal vectors. For large scale Canton Tower, OSP based on generalized genetic algorithm was carried out after the model reduced by model reduction technique and the companions between different reduction methods were conducted (Yi et al. 2012). Then multi-dimensional OSP method (Yi et al. 2013) was also proposed for the Canton Tower. Guyan reduction (Guyan 1965), which is used to reduce the number of degrees of freedom originally, has been also applied for OSP (Penny et al. 1994). Kammer and Peck (2008) investigated an iterative Guyan expansion method for OSP and compared it with Guyan reduction method. Variance method was presented and compared with other five OSP methods in modal identification of a bridge (Meo and Zumpano 2005). Unique characteristic of variance method is that it can be used to determine optimal number of sensors. Cobb and Liebst (1997) introduced an OSP method for the purpose of identifying damaged elements.

Meanwhile, many new algorithms and improved optimal algorithms were presented for improving OSP computational efficiency. Yi et al. (2012) determined the optimum design of 
sensor arrays for parameter identification of Canton Tower by asynchronous-climb monkey algorithm (AMA). Li et al. (2004) developed an efficient method using the uniform design method for OSP which resulted in the reduction of workload above $90 \%$. An improved simulated annealing algorithm was proposed by Tong et al. (2014). Besides, some improved genetic algorithms (GA) were also proposed to improve the convergence speed (Yao et al. 1993, Guo et al. 2004, Liu et al. 2008, Kang et al.2008).

Vibration modes of large scale truss structures are very complicated, such as the jacket offshore platform, the signal tower, Beijing National Stadium and so on. In amount number of degrees of freedom, how to select some degrees of freedom which can reflect structural entirety vibration characteristics and have high signal to noise ratio (SNR) is very important. In addition, for the complicated truss structures, it is difficult to determine reasonable number of sensors only according to engineers' experience.

Therefore, for truss structures, an OSP strategy is presented. The rest of the paper is organized as follows: section 2 describes the proposed OSP strategy in detail, and also presents the procedure of the proposed method. In Section 3, simulation on a scaled jacket offshore platform is conducted to obtain the number of sensors and the corresponding sensor layout. In Section 4, experiment is conducted to verify the effectiveness of the obtained sensor layout in parameter identification. In section 5, the influence of the proposed method using different optimal algorithms and model reduction technique on optimal results is evaluated and compared. In section 6 , conclusions are drawn.

\section{Description of the proposed OSP strategy}

The proposed OSP strategy includes two parts: the OSP method and the optimal number of sensors determination approach with their detail descriptions in section 2.1 and 2.2 respectively. Subsequently, the procedure of the proposed OSP strategy will be introduced in section 2.3.

\subsection{The OSP method}

For engineering structures, a sensor layout should meet many requests in actual measurements to provide the best performance of the structures, such as high SNR of measuring points, integrity and independence property of tested modes and so on. An OSP method based on model reduction technique and structure subsection technique (SST) was proposed. GA was selected to conduct the proposed OSP method, with degrees of freedom set as variables.

In the OSP process, the determination of optimal objective function and the constraint conditions for variables are two critical factors, which have a great influence on the performance of the selected sensor layout. According to the proposed method, the two factors would be processed as follow.

(1) Optimal objective function determination

The purpose of model reduction technique, as originally conceived, was to reduce number of degrees of freedom in a large finite element model to make the solution of the resulting eigenvalue problem more manageable. This leads to degrees of freedom divided into two categories including master degrees of freedom (to be maintained) and slave degrees of freedom (to be removed). Accuracy of model reduction technique is determined by both the number and the locations of master degrees of freedom. So the question arises as to how the number and locations of master 
degrees of freedom are to be determined. According to experience and analysis, some basic principles for the selection of master degrees of freedom are presented, such as,

1. Number of master degrees of freedom is at least twice of interested modes.

2. Direction of structural vibrations should be set as master degree of freedom.

3. Locations with large mass and small stiffness should be selected as master degrees of freedom and so on.

It is seen that the reasonable master degrees of freedom are able to reflect structural entirety vibration characteristics and have high kinetic energy. In many respects, the criterion for choosing locations of sensors in a large scale structure is the same with qualities of master degrees of freedom. Therefore, OSP process can be treated as the process of selecting of an optimal group of master degrees of freedom for model reduction technique. By setting the optimal group of master degrees of freedom as locations of sensors, the obtained sensor layout can display good characteristics that master degrees of freedom have, such as high SNR, the ability of representing structural entirety vibration characteristics and so on.

In order to evaluate the effectiveness of the selected master degrees of freedom, the difference between natural frequency $f$ and standard natural frequency $F$ is set as optimal objective function for GA, shown in Eq. (1).

$$
\operatorname{Obj} V=\sum\left|f_{t}-F_{t}\right|
$$

where, $f_{t}$ is the $t$-th natural frequency obtained by modal analysis according to the reduced mass and stiffness. $F_{t}$ is the $t$-th natural frequency by modal analysis according to the original mass and stiffness.

After calculation, an optimal group of master degrees of freedom, which makes objective function value smallest, can be determined by GA. The selected master degrees of freedom could be set as locations of sensors.

(2) Determination of constraint conditions for variables

For truss structures, when there are no constraint conditions for candidate degree of freedom variable, the optimal sensor layout got by optimization calculation may focus on some local substructures, resulting in damage in other substructures not identified. To provide constraint conditions for the candidate degree of freedom variable, SST was proposed to guarantee the rationality of the distribution of sensors, with detailed description as follow:

According to vibration characteristics of target modes and given number of sensors $N$, the structure is divided into $N$ subsections with no overlaps between each other to make the distribution of sensors uniform and reasonable. By setting the number of subsections as the number of variables, the range of each variable is in its corresponding subsection, avoiding placing two or more sensors at the same candidate location.

Normally, for different truss structures, the division method of SST would be a few different. For a jacket offshore platform model, the division method would be described in section 3.2.

\subsection{The optimal number of sensors determination}

In modal analysis based on model reduction technique, as the number of master degrees of freedom increases and the locations of master degrees of freedom become more reasonable, the 
optimal objective function value would become smaller. When the number of master degrees of freedom reaches to a certain number $N_{O N S}$, the optimal objective function value would decrease a little as the number increases. It implies $N_{O N S}$ master degrees of freedom have enough ability to represent the structural entirety vibration characteristics. And there is little improvement in description of structural entirety vibration characteristics as the number increases.

Therefore, the optimal number of master degrees of freedom or sensors could be determined according to the trend of the optimal objective function value curve, which is obtained by conducting OSP calculations at different number of sensors. Normally, $N_{O N S}$ is a number in a little range, in which the optimal objective function value tends to converge.

\subsection{The procedure of the proposed OSP strategy}

The procedure of the proposed OSP strategy is shown in Fig. 1, including two parts. The first part is the OSP calculation in case of given number, as shown in pink region. Second part is the optimal number of sensors calculation. The detailed descriptions are as follow.

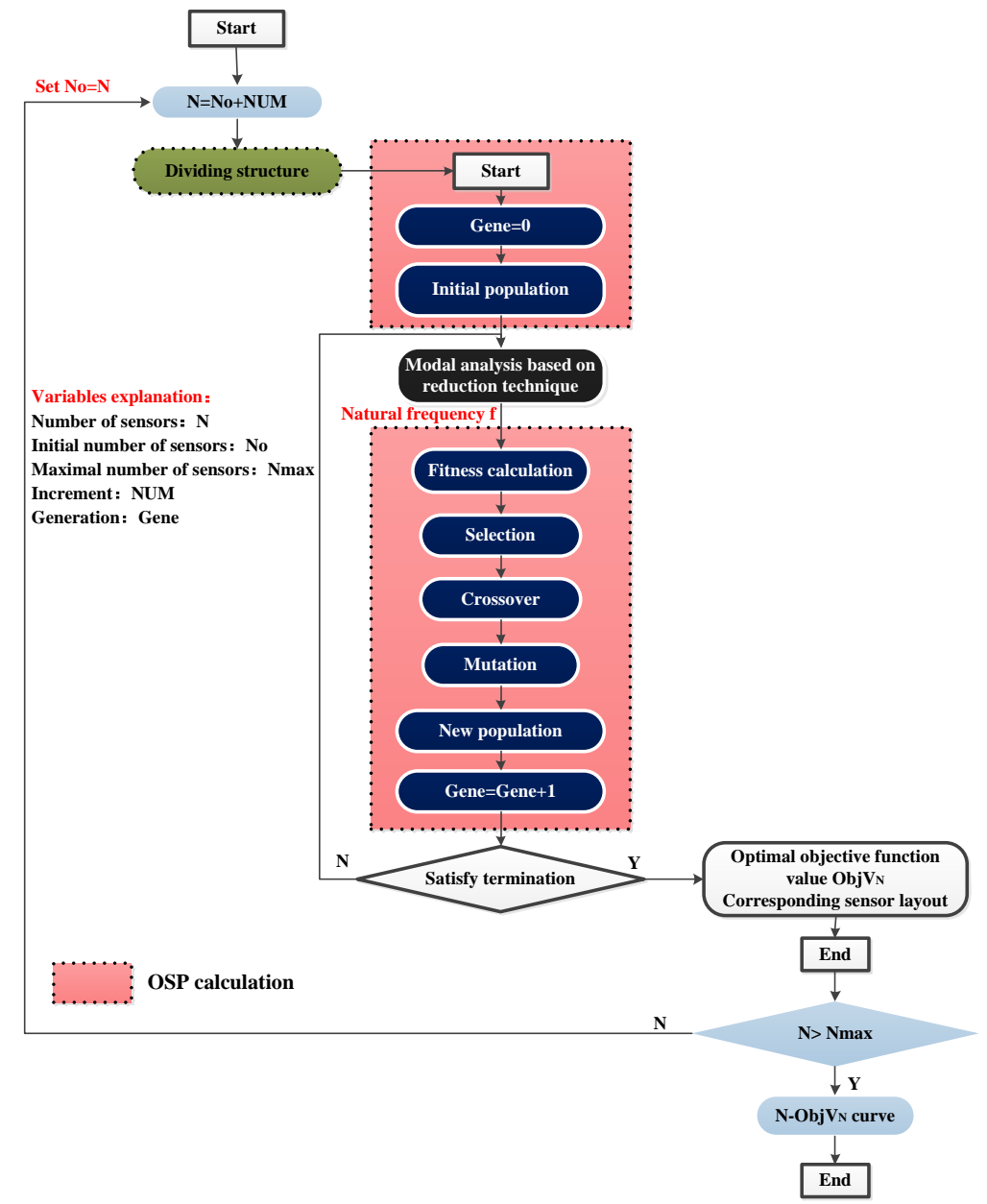

Fig. 1 The procedure of the proposed OSP strategy 


\subsubsection{OSP calculation}

Firstly, when the number of sensors is $N$, the truss structure is divided into $N$ subsections according to vibration characteristics. Then the OSP calculation is conducted as follow:

Step 1: Generate initial population. Each individual has $N$ degrees of freedom.

Step 2: Conduct modal analysis based on model reduction technique.

Step 3: Calculate objective function.

Step 4: Generate new population by selection, crossover and mutation.

Step 5: Repeat step 2 to 4 until satisfy the termination, and output the optimal objective function value $O b j V_{N}$ and the corresponding optimal sensor layout.

In step 2, $N$ degrees of freedom generated in step 1 are set as master degrees of freedom. According to the selected master degrees of freedom, model reduction technique is conducted to get the reduced mass and stiffness. Then, by modal analysis using the reduced mass and stiffness, the natural frequency $f$ could be obtained for objective function calculation. In the step, many model reduction techniques developed can be adopted, such as Guyan reduction method (Guyan 1965), Improved Reduced System (IRS) (O'Callahan 1989), Iterated Improved Reduced System (IIRS) (Friswell et al. 1995), and Kuhar reduced method (Kuhar and Stahle 1974) and so on. Influences of the proposed OSP strategy using different model reduction techniques on optimal results will be discussed in section. 5 .

After the optimization calculation at the given number of sensors $N$, the optimal objective function value $\operatorname{Obj}_{N}$ and corresponding optimal sensor layout would be output.

\subsubsection{Optimal number of sensors calculation}

By setting number of sensors $N$ as variable, the optimal objective function values at different numbers of sensors can be obtained. Subsequently, the optimal number of sensors could be determined according to the characteristic of convergence of the optimal objective function value curve.

\section{Case study}

A scaled jacket offshore platform was adopted to evaluate the effectiveness of the proposed OSP strategy. Firstly, the optimal number of sensors and corresponding optimal sensor layout of the scaled model were obtained by the proposed strategy. Secondly, MKE and maximal non-diagonal element of MAC matrix were used to evaluate SNR and mode independence properties of the optimal sensor layout.

In the case study, the proposed method including SST, Guyan reduction and SGA was applied on the scaled model (called SGA method), with Guyan reduction and SGA conducting modal analysis and optimization calculation respectively.

Guyan reduction technique is expressed as follow 


$$
\begin{gathered}
K \Phi=\omega^{2} M \Phi \\
{\left[\begin{array}{cc}
K_{m m} & K_{m s} \\
K_{s m} & K_{s s}
\end{array}\right]\left[\begin{array}{l}
\Phi_{m} \\
\Phi_{s}
\end{array}\right]=\omega^{2}\left[\begin{array}{cc}
M_{m m} & 0 \\
0 & M_{s s}
\end{array}\right]\left[\begin{array}{l}
\Phi_{m} \\
\Phi_{s}
\end{array}\right]}
\end{gathered}
$$

where $\Phi_{m}$ is the modes of the master degrees of freedom; $K_{m m}, M_{m m}$ are the stiffness and mass matrix of master degrees of freedom respectively; $\Phi_{s}$ is the mode of the slave degrees of freedom; $K_{s s}, M_{s s}$ are the stiffness and mass matrix of slave degrees of freedom respectively, $M_{s s} \approx 0$. Neglecting the inertia terms for the second set of equations gives

$$
K_{s m} \Phi_{m}+K_{s s} \Phi_{s}=0
$$

which is used to eliminate the slave degrees of freedom so that

$$
\Phi=\left[\begin{array}{l}
\Phi_{m} \\
\Phi_{s}
\end{array}\right]=\left[\begin{array}{c}
I \\
-K_{s s}^{-1} K_{s m}
\end{array}\right] \Phi_{m}=T_{s} \Phi_{m}
$$

where $T_{s}$ is the transform matrix. The reduced mass and stiffness matrices are then given by

$$
M_{r}=T_{s}^{T} M T_{s} \quad K_{r}=T_{s}^{T} K T_{s}
$$

where $M_{r}$ and $K_{r}$ are the reduced mass and stiffness matrices. Then the natural frequency $f$ is obtained by modal analysis using the reduced mass and stiffness.

\subsection{The scaled jacket offshore platform model}

Fig. 2 shows the finite element model of the scaled jacket offshore platform with 12 diagonal bracings, 12 horizontal bracings, 4 vertical bracings, a deck plat and 4 bottom supports. The model is $1.56 \mathrm{~m}$ height, with diagonal bracings and horizontal bracings made of $25 \times 25 \times 3 \mathrm{~mm}$ angle iron and vertical bracings made of $40 \times 40 \times 4 \mathrm{~mm}$ angle iron. Dimensions of deck plate and bottom supports are $0.5 \times 0.5 \times 0.008 \mathrm{~m}$ and $0.25 \times 0.25 \times 0.014 \mathrm{~m}$ respectively. The whole model is made of stainless steel with Young's modulus of $195 \mathrm{GPa}$, Poisson ratio of 0.30 , and density of $7850 \mathrm{~kg} / \mathrm{m}^{3}$. It is fixed on the ground by 4 bottom supports. The initial population size NIND is 20 and the maximum number of generations Gene is 30 . The gap GAAP is set as 0.85 , which represents the overlap degree between the parent generation and the offspring generation.

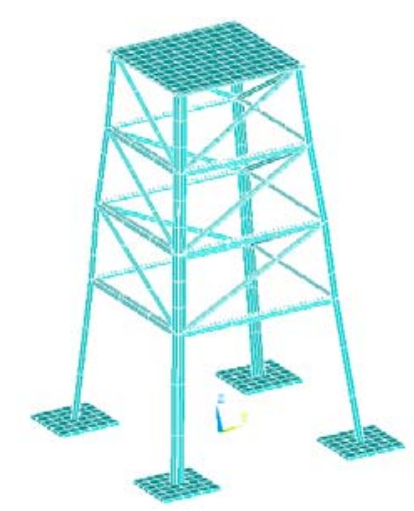

Fig. 2 Finite element model of the scaled jacket offshore platform 
Table 1 First five modes

\begin{tabular}{ccc}
\hline \hline Order of mode & Frequency $(\mathrm{Hz})$ & Mode shape \\
\hline $1^{\text {st }}$ & 27.191 & First bending $\left(\mathrm{X}-45^{\circ}\right.$ direction $)$ \\
$2^{\text {nd }}$ & 27.343 & First bending $\left(\mathrm{X}+45^{\circ}\right.$ direction $)$ \\
$3^{\text {rd }}$ & 42.214 & First torsion \\
$4^{\text {th }}$ & 74.899 & Deck plate vibration \\
$5^{\text {th }}$ & 109.80 & Bracings vibration \\
\hline
\end{tabular}

Table 1 is frequencies and mode shapes of the first five modes obtained from FEM analysis. It is seen that the $1^{\text {st }}, 2^{\text {nd }}, 3^{\text {rd }}$ and $5^{\text {th }}$ modes are global vibrations, and the $4^{\text {th }}$ mode is local vibration. Only global vibration characteristics are chosen as the target modes to be identified. For the large scale jacket offshore platforms, damages on vertical bracings have greater influence on the whole property of the structure than damages on diagonal and horizontal bracings. Therefore only the $1^{\text {st }}$, $2^{\text {nd }}, 3^{\text {rd }}$ and $5^{\text {th }}$ modes are selected.

\subsection{Application of the proposed OSP strategy on the scaled platform}

To identify global vibration modes of the scaled model, sensors would be placed on vertical bracings, which are usually set as major monitoring objects. SST is applied as follow:

1. In order to guarantee integrity of target modes, the number of sensors $N$ was set as $S$ times as number of vertical bracings $M$, where $S$ was an integer larger than 1 .

2. Every vertical bracing was divided into $S$ subsections equally along axial direction. In each subsections, only one sensor was placed.

3 . The number of variables is equal to the number of subsections $(\mathrm{N})$. Degrees of freedom in the $i$-th subsection are set as the search space of the $i$-th variable $(1 \leq \mathrm{i} \leq \mathrm{N})$.

Therefore, the number of sensors on each bracing was same and the distribution of sensors on each bracing was from bottom to top, guaranteeing the rationality of the distribution to some extent. Parameters of the method for the scaled jacket offshore platform were set: initial number of sensor $N_{0}=4$; increment $N U M=4$; maximal number of sensors $N_{M A X}=40$.

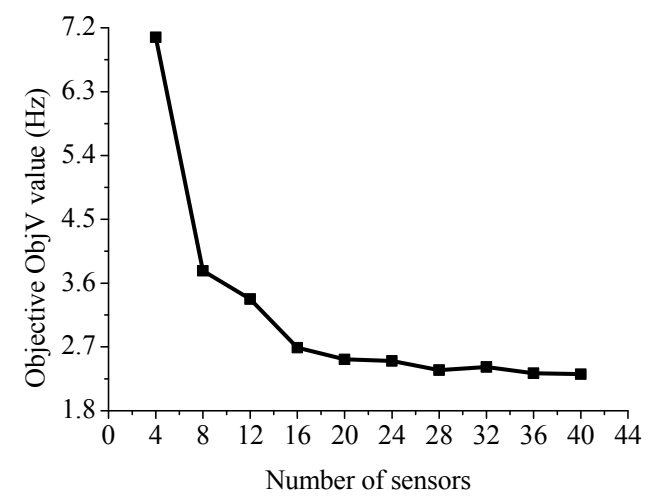

Fig. 3 Number of sensors - Optimal $O b j V$ value curve 


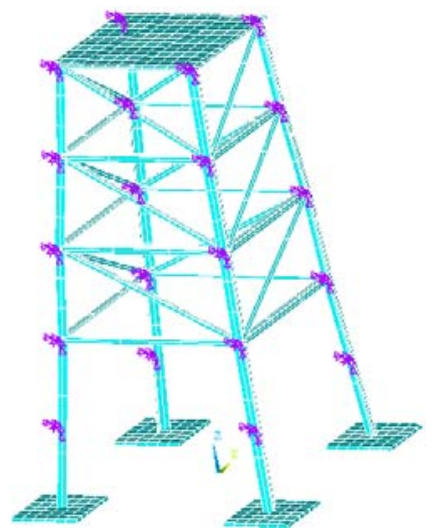

(a) Number of sensors is 20

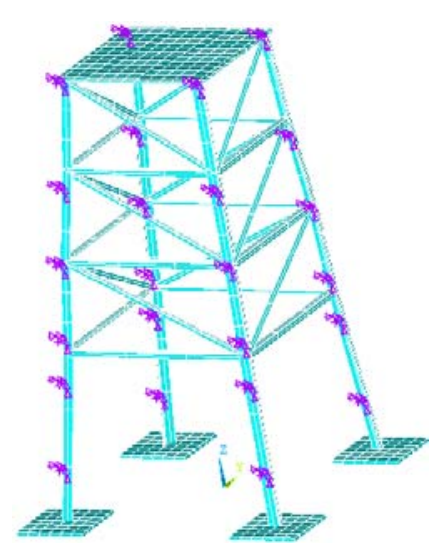

(b) Number of sensors is 24

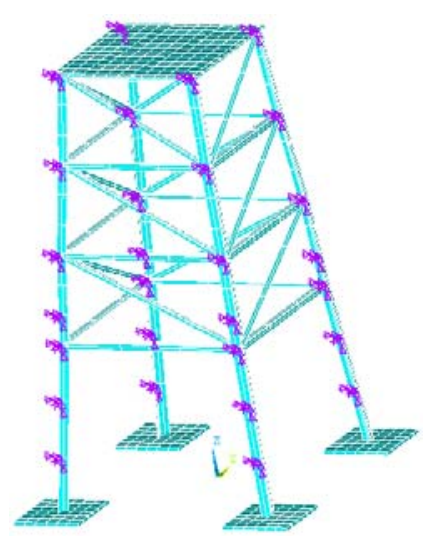

(c) Number of sensors is 28

Fig. 4 Optimal sensor layout of SGA method

Optimal $O b j V$ values at different number of sensors were obtained as shown in Fig. 3. It is seen that when number of sensors $N$ is around 20, optimal $O b j V$ value curve tends to converge.

When number of sensors is 20, 24 and 28, the optimal sensor layouts are shown in Fig. 4. From Fig. 4(a), it is seen that the distribution of 20 sensors covers whole vertical bracings with all 16 bolted connections captured, which is good for obtaining integrity vibration modes and identifying damage like looseness of bolt. Meanwhile, from Figs. 4(b)-4(c), it is observed that the distributions of 24 and 28 sensors also reasonably cover whole vertical bracings with 8 and 16 bolted connections captured respectively.

\subsection{Evaluations of the obtained optimal sensor layout}

Two parameters $E_{N}$ and $M A C_{\max }$ were used to evaluate vibration energy and mode independence property of $N$ sensors, with results shown as Figs. 5(a) and 5(b) respectively.

$E_{N}$ is MKE sum of $N$ selected sensors as expressed in Eq. (6).

$$
E_{N}=\sum_{i=1}^{N} \sum_{t=1}^{T} E_{i t}=\sum_{i=1}^{N} \sum_{t=1}^{T}\left(\Phi_{i t} \sum_{j} M_{i j} \Phi_{j t}\right)
$$

where $E_{i t}$ is MKE of the $i$-th sensor in the $t$-th mode, $\Phi_{i t}$ is the $i$-th sensor corresponding coefficient in the $t$-th mode, $\Phi_{j t}$ is the $j$-th coefficient in the $t$-th mode, $M_{i j}$ is the term in the $i$-th sensor corresponding row and $j$-th column of the finite element model mass matrix, $T=1,2,3,5, N$ is the given number of sensors, from 4 to 40 .

In order to compare average energy of $N$ sensors with average energy of all nodes on 4 vertical bracings, $E_{N T}$ is given as

$$
E_{N T}=N \times E_{\text {Ave }}=N \times\left(E_{\text {Total }} / \text { Total }\right)=N \times\left(\left(\sum_{i=1}^{\text {Total }} \sum_{t=1}^{T} E_{i t}\right) / \text { Total }\right)
$$


where Total is the number of nodes on 4 bracings; $E_{\text {Total }}$ is MKE sum of Total nodes; $E_{\text {Ave }}$ is the average MKE of all nodes on vertical bracings.

$M A C_{\max }$ is maximal non-diagonal element of MAC matrix (Eq. (8)).

$$
M A C_{i j}=\frac{\left(\Phi_{i}^{T} \times \Phi_{j}\right)}{\sqrt{\left(\Phi_{i}^{T} \times \Phi_{i}\right) \times\left(\Phi_{j}^{T} \times \Phi_{j}\right)}}
$$

where $\Phi_{i}$ and $\Phi_{j}$ are the $i$-th and $j$-th modal vector of $N$ sensors respectively.

From Fig. 5(a), it is seen that $E_{N}$ is always higher than $E_{N T}$ at different given number of sensors $N$, verifying sensors obtained by the proposed method have relatively high vibration energy and SNR. From Fig. 5(b), it can be observed that $M A C_{\max }$ at different optimal number of sensors is around 0.04 , satisfying request.

From the distributions of sensors and evaluation results, when $N=20,24$ and 28 , the corresponding optimal sensor layouts got by the proposed OSP method can obtain relatively complete and independent modes and also guarantee high SNR of sensors. The optimal number of sensors can be determined as 20 .

\section{Experiments and results}

\subsection{Experimental set-up and instruments}

Experimental set-up and instruments are shown in Figs. 6 and 7 respectively. CA-YD-103 accelerometer was used to measure acceleration time history and the impulse was applied using a force hammer. Both response and excitation signals were collected and analyzed by DASP software to get mode parameters. Sensors were placed according to the optimal sensor layout when $N=20$.

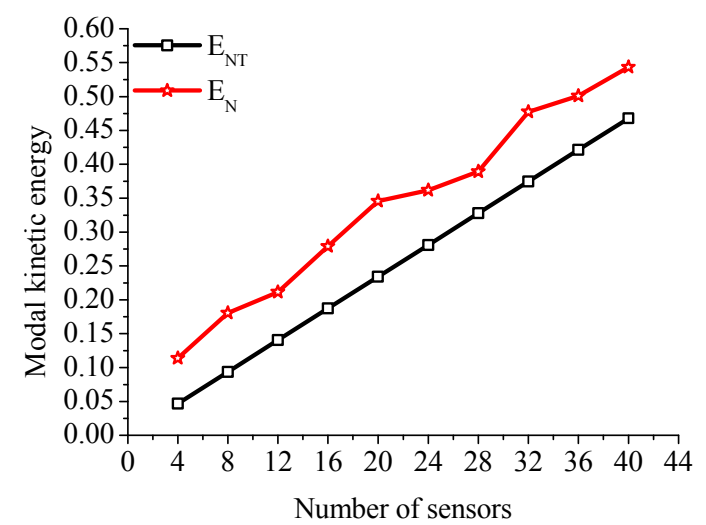

(a) $E_{N}$ results

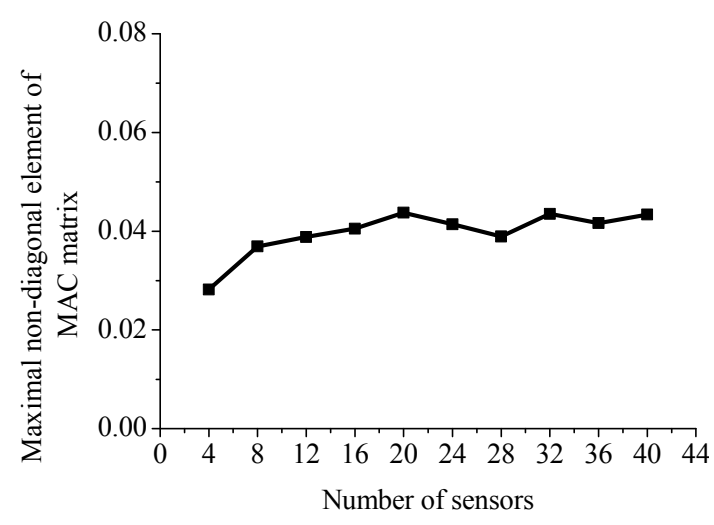

(b) $M A C_{\max }$ results

Fig. 5 Evaluations of proposed method 


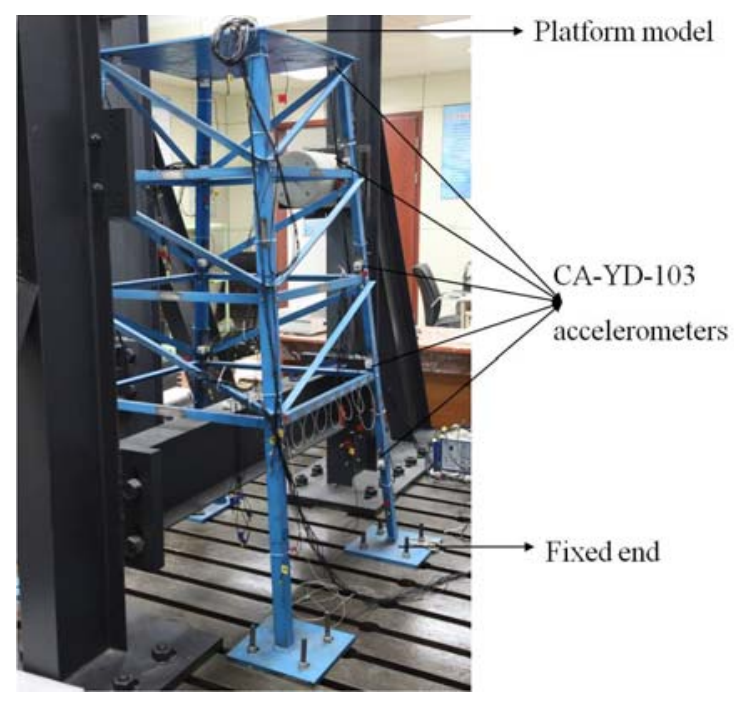

Fig. 6 Experimental setup

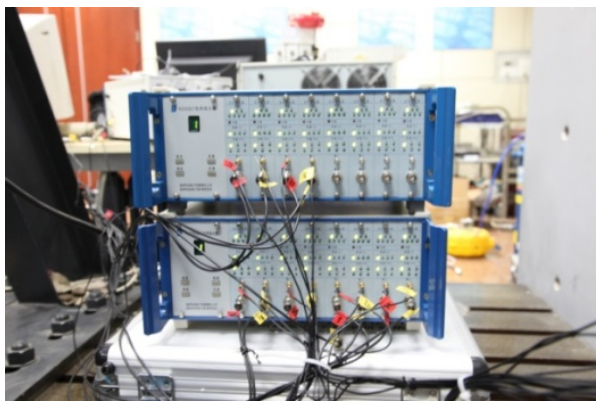

(a) Charge amplifier

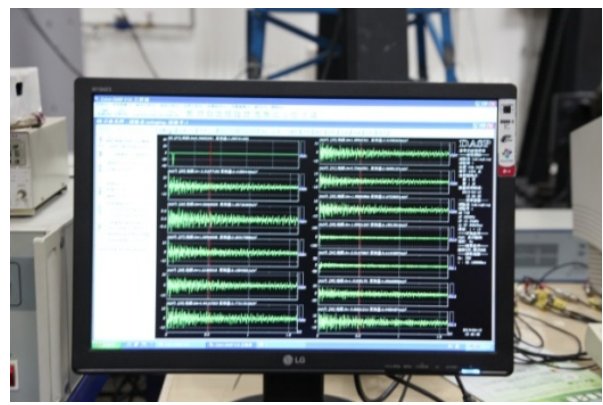

(b) Signal acquisition interface

Fig. 7 Experimental instruments

\subsection{Experimental results}

Frequencies of the $1^{\text {st }}, 2^{\text {nd }}$ and $4^{\text {th }}$ modes are presented in Table 2 along with the results of the finite element analysis. It is seen that both frequencies of simulation and experiment agree reasonably well. MAC matrix of tested modes is shown in Table 3. It is observed that the $M A C_{M A X}$ is 0.056 , satisfying request well.

In order to evaluate the accuracy of the obtained experimental mode parameters, the comparison parameter $C P_{i j}$ was defined,

$$
C P_{i j}=\Phi_{i j} / \phi_{i j}
$$


Table 2 Frequency results

\begin{tabular}{ccccc}
\hline \hline Order of mode & Experiment $(\mathrm{Hz})$ & Simulation $(\mathrm{Hz})$ & Error & Mode shape \\
\hline $1^{\text {st }}$ & 26.087 & 27.191 & $4.06 \%$ & First bending $\left(\mathrm{X}-45^{\circ}\right.$ direction $)$ \\
$2^{\text {nd }}$ & 27.051 & 27.343 & $1.07 \%$ & First bending $\left(\mathrm{X}+45^{\circ}\right.$ direction $)$ \\
$4^{\text {th }}$ & 41.512 & 42.214 & $1.66 \%$ & First torsion \\
\hline
\end{tabular}

Table 3 MAC matrix

\begin{tabular}{cccc}
\hline \hline$M A C_{i j}$ & $1^{\text {st }}$ & $2^{\text {nd }}$ & $4^{\text {th }}$ \\
\hline $1^{\text {st }}$ & 1 & 0.037 & 0.056 \\
$2^{\text {nd }}$ & 0.037 & 1 & 0.055 \\
$4^{\text {th }}$ & 0.056 & 0.055 & 1 \\
\hline
\end{tabular}

where $\varphi_{i j}$ is $i$-th coefficient in the $j$-th tested mode, $\Phi_{i j}$ is numerical mode parameter corresponding to $\varphi_{i j}$. The average comparison value of $j$-th mode, $A C P_{j}$ was expressed

$$
A C P_{j}=\left(\sum_{i=1}^{N}\left|C P_{i j}\right|\right) / N
$$

where $N$ is number of sensors. Modified experimental result, $\Phi_{i j}$

$$
\Phi_{i j}^{\prime}=\phi_{i j} \times A C P_{j}
$$

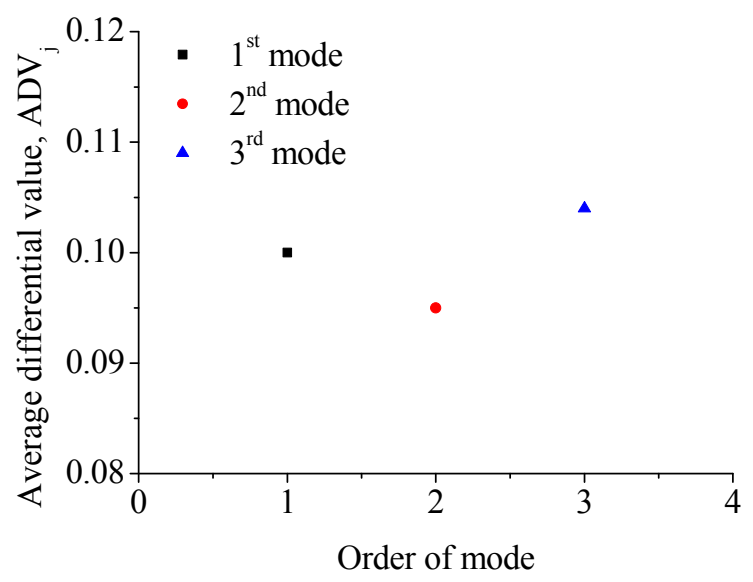

Fig. 8 Average differential values between simulation and experiment 
Then, the average difference ratio of $j$-th mode (Eq. 12) was obtained with results shown in Fig. 8. From Fig. 8, it is seen average difference ratios of all three modes are around 0.1. Although some errors exist, mode shapes between experiment and simulation agree reasonably well.

$$
A D V_{j}=\left(\sum_{i=1}^{N}\left|\left(\Phi_{i j}-\Phi_{i j}^{\prime}\right) / \Phi_{i j}\right|\right) / N
$$

\section{Discussions}

In section 3 and 4, the effectiveness of the proposed strategy based on SST, Guyan reduction and SGA was verified. In this section, different algorithms and model reduction techniques were adopted to investigate their influences on optimal results.

In 1990s, Penny (1994) presented an iterative OSP method based on Guyan reduction with the procedure as follows,

(1) Calculate stiffness $\left(K_{i i}\right)$ mass $\left(M_{i i}\right)$ ratio of $i$-th degree of freedom, $K_{i i} / M_{i i}$.

(2) Delete the degree of freedom with biggest ratio value.

(3) Recalculate stiffness matrix $K$ and mass matrix $M$.

(4) Jump to step1 until reach the given number of sensors.

In order to compare the effectiveness of the iterative reduction approach with GA, the iterative OSP method proposed by Penny was applied in OSP of the scaled model. Meanwhile, the niched genetic algorithm (NGA) was also used, which displays a better performance than SGA.

Moreover, besides Guyan static reduction technique, there are some dynamic reduction techniques including IRS and IIRS, which are more accurate than Guyan reduction. In order to compare with the effectiveness of Guyan reduction in OSP, IRS was also selected to conduct OSP of the scaled model with its transform matrix shown in Eq. (13).

$$
T_{\text {IRS }}=T_{s}+S M T_{s} M_{r}^{-1} K_{s}
$$

where matrix $S$ is expressed in Eq. (14).

$$
S=\left[\begin{array}{cc}
0 & 0 \\
0 & K_{s s}^{-1}
\end{array}\right]
$$

As a whole, there are total 4 different methods, including Penny's iterative method (called Iterative method), the proposed method including SST, Guyan reduction and SGA (called SGA method), the proposed method including SST, Guyan reduction and NGA (called NGA method), the proposed method including SST, IRS and SGA (called IRS method).

Optimal $O b j V$ value curves of the 4 methods are shown in Fig. 9. The right $\mathrm{Y}$ axis is for IRS method and the left one is for the other 3 methods. From Fig. 9, it is seen that optimal ObjV values of IRS method are much smaller than the other 3 methods using Guyan reduction, proving that modal analysis based on IRS is more accurate than Guyan reduction. It is in accordance with reference (Friswell et al. 1995).

Trends of the optimal $O b j V$ curves of the 3 methods using Guyan reduction (Iterative method, SGA method and NGA method) agree reasonably well. The optimal numbers of sensors determined by both SGA and NGA are same around 20 (as red circle shown), and the optimal 
number of sensors got by Iterative method is around 32 (as purple circle). However, the characteristic of convergence of IRS method is worse than the other 3 methods. According to the trend of optimal $O b j V$ value curve of IRS method, $N_{\text {ONS }}$ can be set as 28 .

When $N=20,24$ and 28, optimal sensor layouts of Iterative method, NGA method and IRS method are shown in Figs. 10-12, with optimal results of SGA method shown in Fig. 4.

From Fig. 10, it is observed that the distributions of sensors of Iterative method are unreasonable and some sensors are close with each other (as red circles shown). It is because SST is not adopted in the iterative process. Besides, Iterative method is based on the iterative reduction approach, in which the adjacent iterative steps are greatly dependent on each other, resulting in difficulty of obtaining global optimum results.

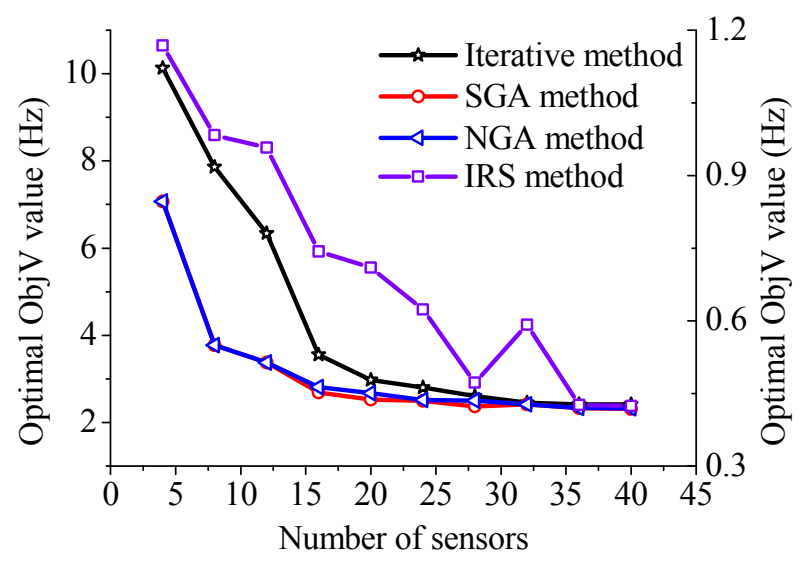

Fig. 9 Number of sensors - Optimal ObjV value curves of 4 methods

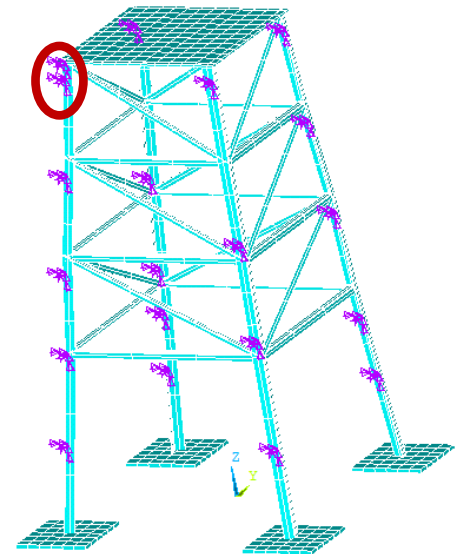

(a) Number of sensors is 20

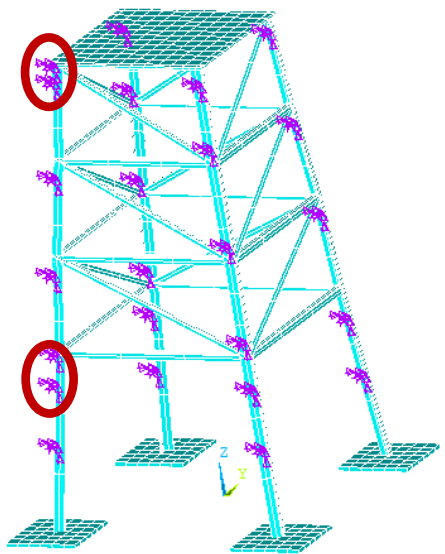

(b) Number of sensors is 24

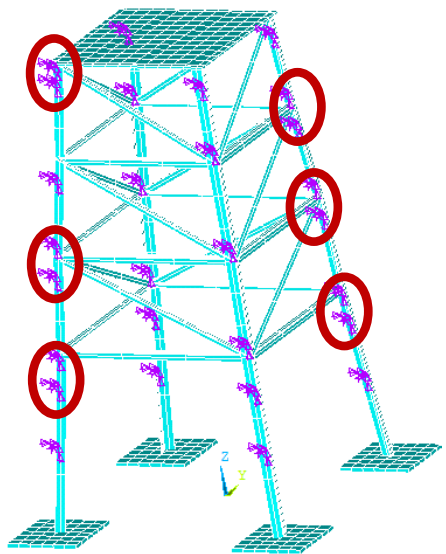

(c) Number of sensors is 28

Fig. 10 Optimal sensor layouts of Iterative method 


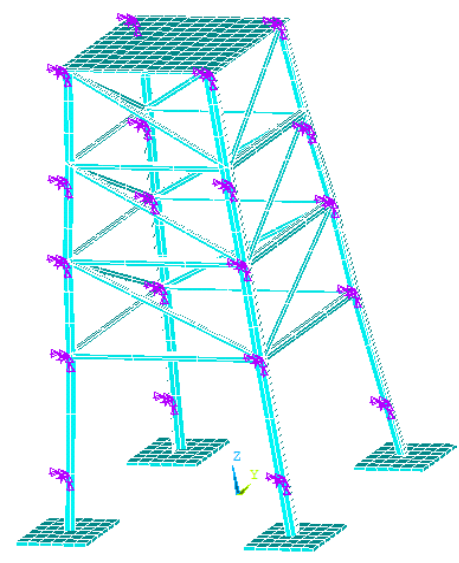

(a) Number of sensors is 20

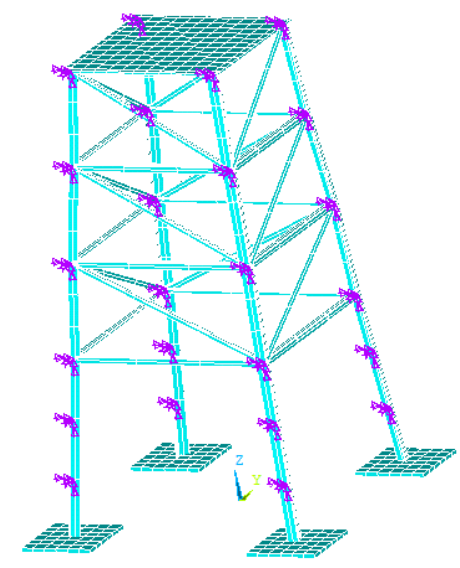

(b) Number of sensors is 24

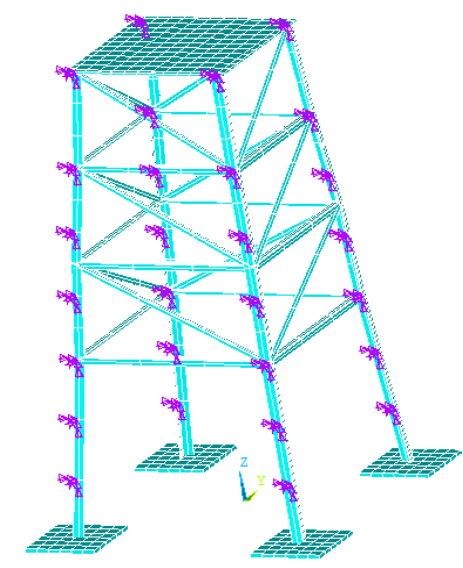

(c) Number of sensors is 28

Fig. 11 Optimal sensor layouts of NGA method

The optimal sensor layouts of the other 3 methods using SST are more reasonable than Iterative method. From Figs. 4, 11 and 12, it is seen that the distributions of sensors could cover whole vertical bracings (except Fig. 12(a)) with bolted connections well captured.

In order to investigate the influence of the proposed method using different model reduction techniques on optimal results, comparisons of optimal sensor layouts obtained by SGA method and IRS method were conducted. From Figs. 4 and 12, it is observed that the distributions of sensors got by SGA method are as good as IRS. When $N=28$, it is seen that the distributions of sensors of both methods are exactly same (as shown in Figs. 4(c) and 12(c)). It shows that the proposed method using different model reduction techniques has little influence on the optimal sensor layouts.

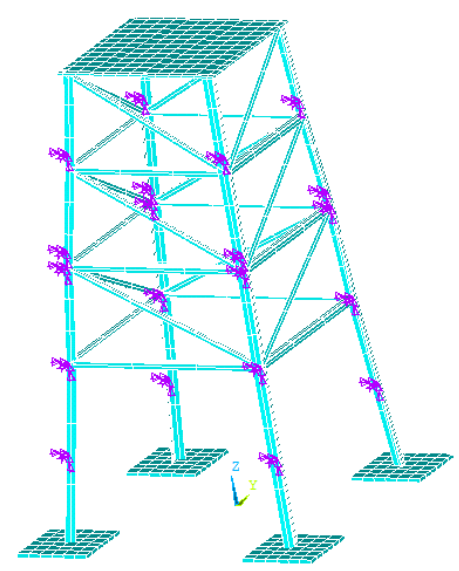

(a) Number of sensors is 20

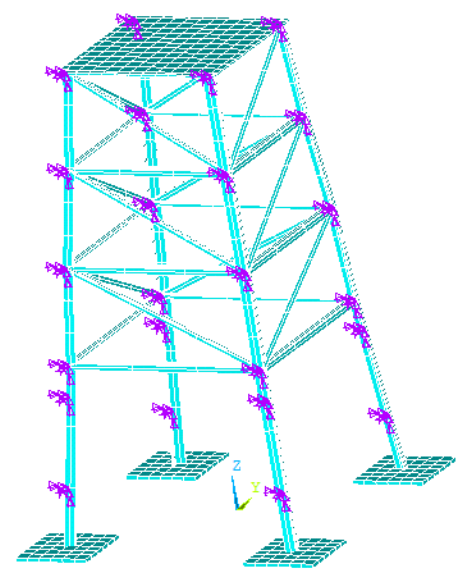

(b) Number of sensors is 24

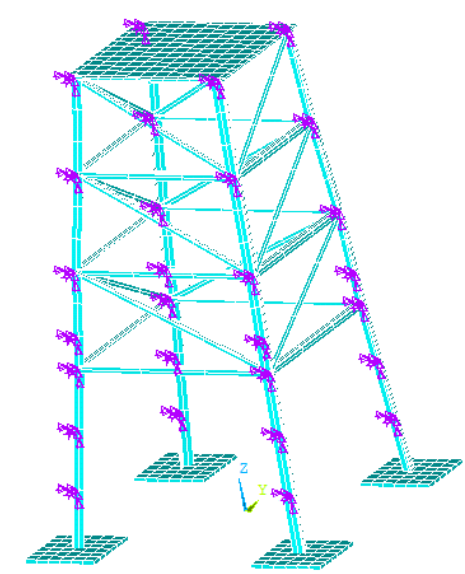

(c) Number of sensors is 28

Fig. 12 Optimal sensor layouts of IRS method 


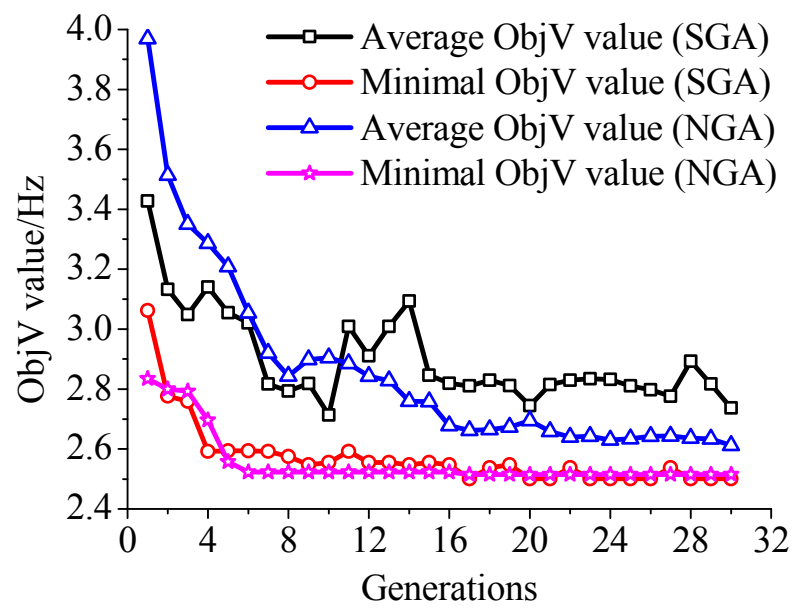

Fig. 13 Optimal function value curves of SGA method and NGA method

In order to investigate the influence of the proposed method using different optimal algorithms on optimal results, comparisons of optimal results got by SGA method and NGA method were conducted. When $N=24$, the optimal $\operatorname{Obj} V$ value curves of both methods are shown in Fig. 13. From Fig. 13, it is observed that optimal $O b j V$ value using NGA quickly tends to a constant and the average optimal $O b j V$ values of NGA method is steadier than SGA method, proving NGA method shows a better characteristic of convergence. From Figs. 4 and 11, it is seen that sensor layouts of both method could cover whole structures and distributions of sensors are all reasonable and acceptable. When $N=20,24$ and 28, there are 12,12 and 20 locations of sensors obtained by SGA method same with NGA method's results. It demonstrates the influence of the proposed method using different optimal algorithms on optimal results is very little.

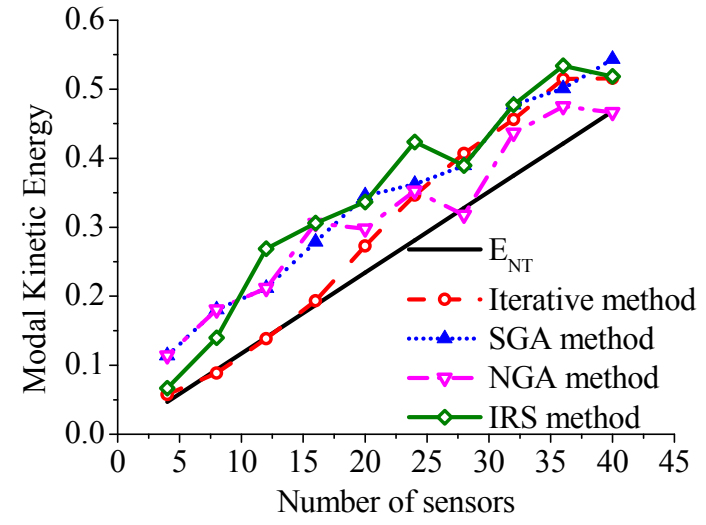

(a) $E_{N}$ results

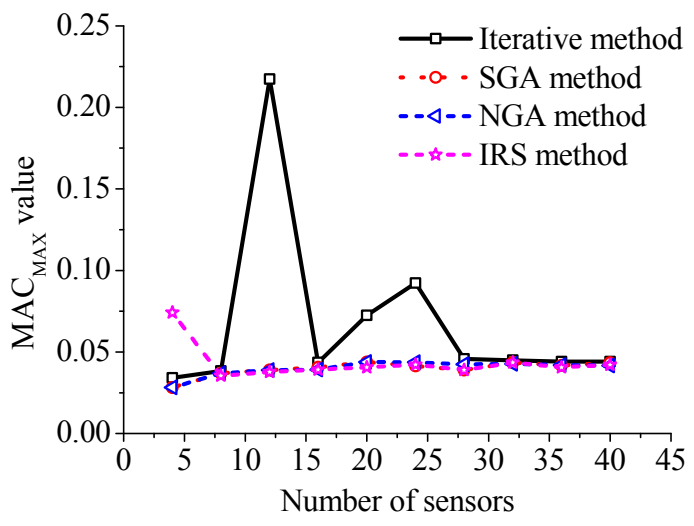

(b) $M A C_{\max }$ results

Fig. 14 Evaluations of the 4 methods 
In order to evaluate the 4 methods quantitatively, the parameters $E_{N}$ and $M A C_{\max }$ were adopted with results shown in Fig. 14. From Fig. 14(a), it is observed that the average vibration energy of sensors obtained by the 4 methods is higher than the average energy of all nodes on vertical bracings, demonstrating that the proposed method using model reduction technique can guarantee high vibration energy of selected locations, which is good for improving SNR.

From Fig. 14(b), at different number of sensors, mode independence property got by SGA method, NGA method and IRS method constantly satisfies request well. However, optimal sensor layouts obtained by Iterative method shows relatively bad independence property at some cases, for example when $N=12, N=24$, demonstrating SST plays an important roles in controlling the distribution of sensors.

\section{Conclusions}

For large scale truss structures, an OSP method using SST, model reduction technique and GA was proposed. According to the optimal $\operatorname{Obj} V$ value curve, an optimal number of sensors determination approach was also presented. Simulation was conducted on a scaled jacket offshore platform to obtain the optimal number of sensors and corresponding sensor layout by the proposed OSP strategy. Subsequently, the experiment was carried out to verify the effectiveness of the optimal sensor layout. Moreover, the influence of the proposed method using different optimal algorithms and reduction techniques on optimal results was discussed. Some conclusions are summarized as follows:

- For large scale truss structures, an OSP method was proposed. Sensor layout obtained by the proposed method has high SNR and maintains good independence between tested modes.

- The method to determine the number of sensors was also proposed for truss structures. The effectiveness of the proposed method has been verified according to the results.

- By comparing Iterative method and the proposed method, it is seen that SST plays an important role in guaranteeing the rationality of the distribution of sensor layout.

- For the scaled jacket offshore platform, it is observed that the influence of the proposed method using different optimal algorithms and model reduction techniques on optimal results is little.

\section{Acknowledgments}

This research work was jointly supported by the National Natural Science Foundation of China(Grant Nos. 11472276 and 11332011) and the State Key Development Program for Basic Research of China (Grant No. 2011CB711100).

\section{References}

Carne, T.G. and Dohmann, C.R. (1995), “A modal test design strategy for modal correlation”, Proceedings of the 13th International Modal Analysis Conference, Schenectady, New York, USA, February. 
Chung, Y.T. and Moore, J.D. (1993), “On-orbit sensor placement and system identification of space station with limited instrumentations", Proceedings of the 11th International Modal Analysis Conference, Kissimmee, Orlando, USA, February.

Cobb, R.G. and Liebst, B.S. (1997), "Sensor placement and structural damage identification from minimal sensor information", AIAA J., 35(2), 369-374.

Friswell, M.I., Garvey, S.D. and Penny, J.E.T. (1995), "Model reduction using dynamic and iterated IRS techniques", J. Sound Vib., 186(2), 311-323.

Guo, H.Y., Zhang, L., Zhang, L.L. and Zhou, J.X. (2004), "Optimal placement of sensors for structural health monitoring using improved genetic algorithms”, Smart Mater. Struct., 13(3), 528-534.

Guyan, R.J. (1965), "Reduction of mass and stiffness matrices", AIAA J., 3(2), 380.

Hanis, T. and Hromcik, M. (2012), "Optimal sensor placement and spillover suppression”, Mech. Syst. Signal Pr., 28, 367-378.

Imamovic, N. (1998), Model validation of large finite element model using test data, Ph.D Dissertation Imperial College, London, UK.

Kammer, D.C. (1991), "Sensor placement for on-orbit modal identification and correlation of large space structures”, J. Guid Control Dynam., 14(2), 251-259.

Kammer, D.C. and Peck, J.A. (2008), "Mass weighting methods for sensor placement using sensor set expansion techniques", Mech. Syst. Signal Pr., 22, 1515-1525.

Kang, F., Li, J.J. and Xu, Q. (2008), "Virus coevolution partheno-genetic algorithms for optimal sensor placement", Adv. Eng. Inform., 22(3), 362-370.

Kuhar, E.J. and Stahle, C.V. (1974), "Dynamic transformation method for modal synthesis", AIAA J., 12(5), 672-678.

Larson, C.B., Zimmerman, D.C. and Marek, E.L. (1994) “A comparison of modal test planning techniques: excitation and sensor placement using the NASA 8-Bay truss", Proceedings of the 12th International Modal Analysis Conference, Hawaii, USA, February.

Li, Z.N., Tang, J. and Li, Q.S. (2004), "Optimal sensor locations for structural vibration measurements", Appl. Acoust., 65(8), 807-818.

Liu, W., Gao, W.C., Sun, Y. and Xu, M.J. (2008), "Optimal sensor placement for spatial lattice structure based on genetic algorithms", J. Sound Vib., 317(1-2), 175-189.

Meo, M. and Zumpano, G. (2005), "On the optimal sensor placement techniques for a bridge structure", Eng. Struct., 27(10), 1488-1497.

O'Callahan, J.C. (1989), “A procedure for an improved reduced system (IRS) model”, Proceedings of the 7th International Modal Analysis Conference, Las Vegas, UAS, January, 17-21.

Papadimitriou, C., Beck, J.L. and Au, S.K. (2000), "Entropy-based optimal sensor location for structural model updating", J. Vib. Control., 6(5), 781-800.

Papadimitriou, C. (2004), "Optimal sensor placement methodology for parametric identification of structural systems”, J. Sound Vib., 278(4-5), 923-947.

Papadimitriou, C. (2005), "Pareto optimal sensor locations for structural identification", Comput. Method. Appl. M., 194(12-16), 1655-1673.

Papadimitriou, C. and Lombaert, G. (2012), "The effect of prediction error correlation on optimal sensor placement in structural dynamics", Mech. Syst. Signal Pr., 28, 105-127.

Penny, J.E.T., Friswell, M.I. and Garvey, S.D. (1994), "The automatic choice of measurement locations for dynamic testing", AIAA J., 30-36.

Salama, M., Rose, T. and Garba, J. (1987), "Optimal placement of excitations and sensors for verification of large dynamical systems", Proceedings of the 28th Structures, Structural Dynamics, and Materials Conference, Monterey, California, USA, April.

Stephan, C. (2012), "Sensor placement for modal identification”, Mech. Syst. Signal Pr., 27, 461-470.

Tong, K.H., Bakhary, N., Kueh A.B.H. and Yassin A.Y.M. (2014), "Optimal sensor placement for mode shapes using improved simulated annealing”, Smart Struct. Syst., 13(3), 389-406.

Ye, S.Q., Ni, Y.Q. (2012), "Information entropy based algorithm of sensor placement optimization for structural damage detection", Smart Struct. Syst., 10(4-5), 443-458. 
Application of model reduction technique and structural subsection technique on optimal sensor... 373

Yi, T.H., Li, H.N. and Gu, M. (2012), "Sensor placement for structural health monitoring of Canton Tower", Smart. Struct. Syst., 10(4-5), 313-329.

Yi, T.H., Li, H.N. and Wang, X. (2013), "Multi-dimensional sensor placement optimization for Canton Tower focusing on application demands", Smart. Struct. Syst., 12(3-4), 235-250.

Yi, T.H., Li, H.N. and Gu, M. (2011), "Optimal sensor placement for structural health monitoring based on multiple optimization strategies”, Struct. Des. Tall Spec., 20(7), 881-900.

Yi, T.H., Li, H.N. and Zhang, X.D. (2012), "Sensor placement on Canton Tower for health monitoring using asynchronous-climb monkey algorithm”, Smart Mater. Struct., 21(12), 1-12. 